\title{
Bone marrow aspirate microscopy v. bone marrow trephine biopsy microscopy for detection of Mycobacterium tuberculosis infection
}

\author{
Q Sedick, MB ChB, FCPath (Haem), MMed (Haem); J Vaughan, MB ChB, FCPath (Haem), MMed (Haem); \\ T Pheeha, MB ChB, FCPath (Haem), MMed (Haem); N A Alli, MB ChB, FCPath (Haem)
}

Haematopathology Department, National Health Laboratory Service, Chris Hani Baragwanath Hospital, Johannesburg, South Africa

Corresponding author: Q Sedick (docqanita@gmail.com)

\begin{abstract}
Background. Tuberculosis (TB) remains a global health problem. According to the 2013 Global Report on Tuberculosis, 8.6 million people developed TB in 2012 and 1.3 million died from the disease. An estimated 13\% of people who developed TB in 2012 were HIV-positive, and $75 \%$ of these lived in Africa. While pulmonary TB is the commonest form of Mycobacterium tuberculosis infection, extrapulmonary TB is increasingly being detected in HIV-positive patients. Definitive diagnosis of disseminated TB is a challenge owing to atypical presentations and diagnostic difficulties (negative chest radiograph and sputum microscopy and culture). A rapid diagnosis of disseminated TB is desirable, as early initiation of treatment can reduce mortality. Although TB culture is the gold standard for diagnosis of TB, it has a long turnaround time (up to 6 weeks).

Objectives. To identify a potentially faster and more effective diagnostic strategy for disseminated TB.

Methods. A retrospective 18-month review, conducted at a tertiary hospital, comparing histological findings of an auramine O-stained bone marrow aspiration (BMA) smear and a bone marrow trephine (BMT) biopsy specimen with the gold standard of TB culture.

Results. Microscopic examination of BMA smears and BMT biopsy specimens offers a rapid diagnostic strategy, with results available on the same day for the former and within 4 days for the latter. BMT histological examination had a significantly higher detection rate than BMA auramine $\mathrm{O}$ staining compared with TB culture.

Conclusion. We recommend that BMT biopsies remain an essential part of the diagnostic work-up for disseminated TB.
\end{abstract}

S Afr Med J 2015;105(9):773-775. DOI:10.7196/SAMJnew.8171

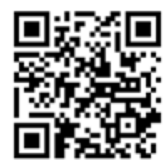

Tuberculosis (TB) remains a significant global health problem. According to the 2013 Global Tuberculosis Report, an estimated 8.6 million people developed TB in 2012 and 1.3 million died from the disease..$^{[1]}$ An estimated 1.1 million (13\%) of the 8.6 million people who developed TB in 2012 were HIV-positive, $75 \%$ of whom were from Africa. In 2012, approximately 450000 people developed multidrug-resistant tuberculosis, of whom an estimated 170000 died. The majority of cases worldwide in 2012 were in South-East Asia (29\%), Africa (19\%) and the Western Pacific region (19\%). ${ }^{[1]}$ The TB incidence rate in South Africa (SA) is among the highest in the world, currently exceeding 1000 cases per 100000 people. ${ }^{[1,2]}$ The predominant age group afflicted with TB is the second and third decades, with devastating socioeconomic implications.

The diagnosis of TB currently includes a detailed medical history, clinical examination, and radiological, microbiological, immunological, molecular and histological investigations. Culture-based detection of Mycobacterium tuberculosis (MTB) in sputa has remained the gold standard of diagnosis until recently, and sputum microscopy for acidfast bacilli (AFB) is the most commonly employed method of screening in the SA setting. However, culture of MTB takes an average of 2 or more weeks, and only $44 \%$ of all new cases ( $15-20 \%$ of children) are actually identified through the presence of AFB on sputum smears. ${ }^{[3]}$ In the setting of TB and HIV co-infection, the sensitivity of sputum smear microscopy is even lower $(\sim 35 \%) .{ }^{[3]}$ The high false-negative rate causes delays in diagnosis and therapeutic intervention, and may lead to further spread of the disease.

Definitive diagnosis of disseminated TB poses a challenge owing to atypical clinical presentations, and often a negative chest radiograph and negative findings on sputum microscopy and culture. This necessitates invasive procedures such as bone marrow aspiration (BMA), bone marrow trephine (BMT) biopsy, soft-tissue biopsy, lumbar puncture, etc.

TB culture is considered to be the gold standard for definitive diagnosis, but the turnaround time is undesirably long (up to 6 weeks), which has an adverse impact on infection control. ${ }^{[3]}$ Microscopic examination of the BMA smears and BMT biopsy specimens offers a far more rapid diagnostic strategy, where results can be available on the same day for the former and within 4 days for the latter. The diagnosis of TB in the bone marrow is made microscopically by the identification of granulomas and/or AFB by Ziehl-Neehlsen (ZN) staining in the BMT biopsy sections. ZN staining is not currently routinely performed on BMA samples owing to its low sensitivity. However, auramine $\mathrm{O}$ staining is reportedly more sensitive than $\mathrm{ZN}$ in sputum, ${ }^{[4]}$ and may offer a higher yield in BMA samples as well.

\section{Objective}

To compare the diagnostic yield for TB between the BMA smear and BMT biopsy specimen against TB culture as the gold standard, and thereby identify a potentially faster and more effective diagnostic strategy for a resource-poor setting.

\section{Methods}

A retrospective 18-month review (January 2009 - June 2010) of bone marrow MTB test results was conducted at Chris Hani Baragwanath Hospital, a tertiary level hospital in Johannesburg, SA. The study was approved by the Human Research Ethics Committee 
of the University of the Witwatersrand (ethics number: M090688). The study population comprised 410 adult and paediatric patients who had a BMA smear and BMT biopsy specimen submitted for analysis to exclude MTB infection. Clinical data were obtained from the bone marrow request forms completed by clinicians, and results were retrieved from the laboratory information system (DisaLab version 04.16.04.373). BMA slides from BMA samples submitted to the National Health Laboratory Service (NHLS) for TB testing were stained with auramine $\mathrm{O}$. The results of the latter were compared with those of BMT biopsy specimen microscopy and BMA TB culture.

BMA smears were stained with Giemsa for conventional microscopy and with auramine $\mathrm{O}$ for detection of AFB using fluorescent microscopy. BMT sections were stained with haemotoxylin and eosin for conventional microscopy. The presence of granulomas necessitated further staining for detection of infective organisms at the discretion of the reporting pathologist. TB culture was done on BMA samples inoculated into special Myco-Flytic culture vials during the BMA procedure. These were then incubated in the Bactec 9240 automated system and inspected weekly for 6 weeks.

The diagnosis of bone marrow TB was made on the basis of one or more of the following criteria: $(i)$ positive TB culture of bone marrow; (ii) detection of granulomas and/or AFB on the BMT biopsy specimen; and (iii) detection of AFB on the BMA sample using the auromine-O staining method.

Exclusion criteria were: $(i)$ samples not inclusive of all test methods, viz. BMA auramine $\mathrm{O}$ staining, BMT biopsy specimen staining and TB culture; and (ii) suboptimal quality samples.

\section{Statistical analysis}

Statistical analysis was performed using STATISTICA software, version 12.0 (Stat Soft (Pty) Ltd). Data are presented as medians (interquartile range (IQR)) and proportions, as appropriate. Sensitivity and specificity were calculated for each test method using TB culture as the gold standard. The Mann-Whitney $U$-test was used to compare continuous variables of interest. The level of statistical significance was taken as $p \leq 0.05$.

\section{Results}

\section{Characteristics of the cohort}

Results from a total of 410 patient samples were analysed (Table 1).

\section{Characteristics of patients diagnosed with tuberculosis}

Out of the 410 patient samples, 123 were positive for TB. Of the samples in which a BMA/BMT biopsy was performed specifically to investigate for $\mathrm{TB}, 32.5 \%$ were positive for $\mathrm{TB}$. The remainder of the patients with TB-positive samples had cytopenias (57.7\%) and haematological malignancies $(0.95 \%)$ (Table 2 ).

Of the 123 samples that were positive for TB, 7 (5.7\%) were diagnosed on BMA auramine O staining, 97 (78.9\%) had granulomas on the BMT biopsy specimen, and 63 (51.2\%) were positive on TB culture. ZN staining was performed in only 90 cases (73.2\%), and was found to be positive for AFB in 38 (42.2\%). Sensitivity and specificity results for the various methods are set out in Table 3 . The organism identified on TB culture was MTB in 58 (92\%) of cases, and M. avium intracellulare complex in the remaining 5. All the BMA auramine $\mathrm{O}$-positive cases with a positive $\mathrm{TB}$ culture were positive for MTB.

Of the 347 patients with samples negative for TB culture (84.6\%), $83(23.9 \%)$ had TB diagnosed by examination of BMA/BMT biopsy
Table 1. Indications for bone marrow examination

\begin{tabular}{ll}
\hline Indications for bone marrow examination & Requests, $\boldsymbol{n}(\%)$ \\
\hline Peripheral cytopenias & $187(45.6)$ \\
$\begin{array}{l}\text { Haematological malignancies (lymphoma and } \\
\text { leukaemia) }\end{array}$ & $158(38.5)$ \\
Suspected disseminated TB & $65(15.8)$
\end{tabular}

\section{Table 2. Characteristics of the cohort}

\begin{tabular}{|c|c|}
\hline Total sample size, $N$ & 410 \\
\hline Adults ( $>18$ years), $n$ & 355 \\
\hline Paediatric patients ( $<18$ years), $n$ & 55 \\
\hline HIV-positive patients in cohort, $n / N(\%)^{*}$ & $251 / 330(76.1)$ \\
\hline Patients diagnosed with $\mathrm{TB}^{\dagger}$ & $123(30.0)$ \\
\hline Patients with peripheral cytopenia/s, $n / N(\%)^{*}$ & $71 / 123(57.7)$ \\
\hline $\begin{array}{l}\text { Patients with clinical suspicion of disseminated TB, } \\
n / N(\%)^{\ddagger}\end{array}$ & $40 / 123(32.5)$ \\
\hline $\begin{array}{l}\text { Patients with suspected haematological } \\
\text { malignancies, } n / N(\%)^{\ddagger}\end{array}$ & $12 / 123(9.8)$ \\
\hline $\begin{array}{l}\text { *Of patients tested for HIV. } \\
\text { 'According to the inclusion and exclusion criteria listed under 'Methods'. } \\
\text { "Of the total number of TB-positive patients. }\end{array}$ & \\
\hline
\end{tabular}

Table 3. Sensitivity and specificity of method against TB culture as reference method

\begin{tabular}{lll}
\hline Test & Sensitivity, \% & Specificity, \% \\
\hline TB culture & 100 & 100 \\
BMT granuloma detection & 56 & 82 \\
ZN staining for AFB & 46 & 94 \\
BMA auramine O staining & 9.3 & 99
\end{tabular}

Table 4. Relationship between TB culture (gold standard) and other methods (BMA auramine $O$ stain, BMT ZN stain and BMT granulomas)

Diagnostic method positive

TB culture negative, $N=347$ $(84.6 \%), n(\%)$

$\begin{array}{ll}\text { Auramine O stain (BMA) positive } & 1(0.3) \\ \text { Granuloma (BMT) positive } & 61(17.6) \\ \text { ZN stain positive } & 21(6.1)\end{array}$

$\mathrm{ZN}$ stain positive

TB culture positive, $N=63$ (15.4\%), $n$ (\%)

Diagnostic method negative

$56(88.9)$

Auramine O stain (BMA) negative

28 (44.4)

Granuloma (BMT) negative

20 (31.7)

specimens. This is an interesting finding with respect to TB culture being the gold standard. In contrast, of the 63 patients with positive TB culture results, 56 (88.9\%) had a negative BMA auramine O stain, 28 (44.4\%) had no granulomas on the BMT biopsy specimen, and 20 (of an available 37) (54.1\%) were negative for AFB on $\mathrm{ZN}$ staining (Table 4).

In order to assess the value added by each of the four methods, test positivity rates in isolation were calculated, viz. TB culture $n=24$ 
Table 5. TB detection rates in HIV-positive patients

\begin{tabular}{llll}
\hline & $\begin{array}{l}\text { Tested for } \\
\text { TB, } \boldsymbol{N}\end{array}$ & $\begin{array}{l}\text { Positive for } \\
\text { TB, } \boldsymbol{n}(\%)\end{array}$ & $\begin{array}{l}\text { CD4 count } \\
\text { (cells/ } \boldsymbol{\mu L})(\boldsymbol{n}=\mathbf{9 9}), \\
\text { median }(\mathbf{I Q R})\end{array}$ \\
\hline HIV-positive patients tested for TB & 251 & $113(45.0)$ & $33(11-95)$ \\
TB culture method & 113 & $58(51.3)$ & $27(9-82)$ \\
BMT granuloma detection & 113 & $88(77.9)$ & $33(14-81)$ \\
ZN staining for AFB (BMT) & 83 & $36(43.4)$ & $28(6-76)$ \\
BMA auramine O staining & 113 & $7(6.2)$ & $7(4-16)$
\end{tabular}

(38.1\%), BMT granulomas $n=38$ (39.2\%), BMT ZN stain $n=0(0 \%)$, BMA auramine O stain $n=0(0 \%)$.

\section{Characteristics of TB patients with HIV}

Of the 330 patients tested for HIV, 251 (76.1\%) were HIV-positive. The number of dual HIV- and TB-positive patients was $113 / 251$ (45.0\%). The sensitivity of TB detection in HIV-positive patients was best with BMT granuloma detection (56.9\%) when compared with $\mathrm{ZN}$ staining (48.5\%) and BMA auramine $\mathrm{O}$ staining (10.3\%) against the gold-standard TB culture. In HIV-positive patients, the median CD4 count was significantly lower in patients with TB than in those without TB: 33 cells $/ \mu \mathrm{L}$ (range 11 - 95) and 117 cells $/ \mu \mathrm{L}$ (range $41-221)$, respectively $(p<0.0001)$. Furthermore, the median CD4 count in patients with positive BMA auramine $\mathrm{O}$ staining was significantly lower $(p<0.0001)$ than that in patients diagnosed with TB by any other modality, viz. 7 cells $/ \mu \mathrm{L}$ (range 4 - 16) and 35 cells $/ \mu \mathrm{L}$ (range $15-98$ ), respectively $(p=0.022)$ (Table 5$)$.

\section{Discussion}

TB remains a significant health problem, the detection of which requires prompt diagnosis. TB culture-based algorithms for diagnosis are relatively expensive ${ }^{[5]}$ and have an unacceptably long turnaround time. Current rapid methods quoted in the literature include rapid liquid culture systems, ${ }^{[3]}$ nucleic acid amplification tests ${ }^{[6]}$ and the microscopic observation susceptibility assay. ${ }^{[7]}$ Studies have also recently shown that in HIV-positive patients with CD4 cell counts $<100$ cells $/ \mu \mathrm{L}$, the TB lipoarabinomannan urine test can detect over half of culture-positive TB patients in less than 30 minutes. $^{[8]}$ In the SA setting, the GeneXpert MTB/RIF assay has become widely available, and reportedly performs better than smear microscopy where TB and HIV are both highly endemic. ${ }^{[9]}$ However, these tests are generally expensive and require specialised equipment and expertise, and to date none has been validated on BMA and BMT biopsy samples. In this study, we evaluated the role of BMT biopsy specimen examination and BMA auramine $\mathrm{O}$ staining in reducing the time taken to establish a diagnosis of disseminated $\mathrm{TB}$, using $\mathrm{TB}$ culture as the gold standard.

Over $95 \%$ of the patients with disseminated TB were HIV-positive, with a median CD4 count of 33 cells $/ \mu \mathrm{L}$. Approximately a third of these patients had a BMA/BMT biopsy performed to investigate for possible TB, and over half had unexplained cytopenias. The sensitivity for detecting TB was highest for histological examination of BMT biopsy specimens (56\%), followed by ZN staining $(46 \%)$, and least sensitive for auramine O staining of BMA samples (9.3\%). Interestingly, the median CD4 counts in HIV-positive patients with TB diagnosed by auramine $\mathrm{O}$ staining of BMA samples were significantly lower than those in patients diagnosed with the other test methods, as a consequence of immune compromise. Although the BMA auramine $\mathrm{O}$ method is rapid and showed excellent specificity, its sensitivity is too low for it to be advocated for routine practice. The reasons for the poor yield using this method are likely to be multifactorial, including poor quality of BMA samples, reduced macrophage activity on the BMA smear and overall poor sensitivity of the test method.

The utility of BMT biopsy specimen examination in the setting of HIV/ TB co-infected patients was reported on previously by Akpek et al., ${ }^{[10]}$ who showed that although the diagnostic sensitivity of bone marrow cultures was equal to that of blood cultures, histological examination of bone marrow resulted in rapid identification of nearly one-third of infected patients who underwent bone marrow examination, and that infection was identified in some patients who were culture-negative. In our study, examination of BMT biopsy specimens proved highly valuable; approximately half possibly reflecting a higher TB bacillary load of the cases of TB were diagnosed microscopically without a positive TB culture, and the diagnosis was expedited in over $60 \%$ of patients in whom the TB culture was positive. Nevertheless, over one-third of patients with a positive TB culture had no bone marrow morphological abnormalities, highlighting the potential utility of molecular techniques in this setting.

\section{Conclusion}

We found that histological examination of BMT biopsy specimens had a significantly higher detection rate than auramine $\mathrm{O}$ staining of BMA samples, compared with the gold-standard TB culture in our setting, where there is a high HIV burden. We recommend that BMT biopsies remain an essential part of the diagnostic work-up for disseminated TB. However, as microscopic examination failed $(p<0.0001)$ to detect a substantial proportion of cases in whom TB culture was positive, other rapid molecular techniques such as GeneXpert are required.

Acknowledgement. The authors thank the Chris Hani Baragwanath Hospital NHLS laboratory staff.

\section{References}

1. Zumla A, George A, Sharma V, Herbert N, Baroness Masham of Ilton. WHO's 2013 global report on tuberculosis: Successes, threats, and opportunities. Lancet 2013;382(9907):1765-1767. [http://dx.doi.org/10.1016/S0140-6736(13)62078-4]

2. World Health Organization (WHO). Global Tuberculosis World Health Organization (WHO). Global Tuberculosis Report 2013. Geneva: WHO, 23 October 2013. http://apps.
who.int/iris/bitstream/10665/91355/1/9789241564656_eng.pdf who.int/iris/bitstream/106
(accessed 10 August 2015).

3. Lange C, Mori T. Advances in the diagnosis of tuberculosis. Lange C, Mori T. Advances in the diagnosis of tuberculosis.
Respirology 2010;15(2):220-240. [http://onlinelibrary.wiley.com/ doi/10.1111/j.1440-1843.2009.01692.x/abstract ]

4. Hooja S, Pal N, Malhotra B, Goyal S, Kumar V, Vyas L. Comparison of Ziehl Neelsen \& auramine O staining methods on direct and concentrated smears in clinical specimens. Indian J Tuberc 2011;58(2):72-76. [http://tbassnindia.org/forms/ IJT_12.pdf]

5. Yakhelef $\mathrm{N}$, Audibert M, Varaine $\mathrm{F}$, et al. Is introducing rapid culture into the diagnostic algorithm of smearnegative tuberculosis cost-effective? Int J Tuberc Lung Dis 2014;18(5):541-546. [http://dx.doi.org/10.5588/ijtld.13.0630]

6. Adelman MW, Kurbatova E, Wang YF, et al. Cost analysis of a

6uclect nucleic acid amplification test in the diagnosis of pulmonary tuberculosis at an urban hospital with a high prevalence of TB/
HIV. PloS One 2014;9(7):e100649. [http://dx.doi.org/10.1371/ journal.pone.0100649]

journal.pone.0100649]
7. Solomon S, Balakrishnan P, Vignesh R, et al. A rapid and lowSolomon S, Balakrishnan P, Vignesh R, et al. A rapid and low-
cost microscopic observation drug susceptibility assay for detecting TB and MDR-TB among individuals infected by HIV in South India. Indian J Med Microbiol 2013;31(2):130-137. [http://dx.doi.org/10.4103/0255-0857.115225]

8. Lawn SD, Kerkhoff AD, Burton R, Meintjes G. Underestimation of the incremental diagnostic yield of HIV-associated tuberculosis in studies of the Determine TB-LAM Ag urine assay. AIDS 2014;28(12):1846-1848. [http://dx.doi.org/10.1097/ QAD.0000000000000305]

9. O'Grady J, Bates M, Chilukutu L, et al. Evaluation of the Xpert MTB/RIF assay at a tertiary care referral hospital in a setting MTB/RIF assay at a tertiary care referral hospital in a setting where tuberculosis and HIV infection are highly endemic. Clin
Infect Dis 2012;55(9):1171-1178. [http://dx.doi.org/10.1093/ cid/cis631]

10. Akpek G, Lee SM, Gagnon DR, Cooley TP, Wright DG. Bone marrow aspiration, biopsy, and culture in the evaluation of HIVinfected patients for invasive mycobacteria and histoplasma infections. Am J Hematol 2001;67(2):100-106. [http://dx.doi. org/10.1002/ajh.1086]

Accepted 30 June 2015. 